\title{
Injection therapy for chronic prostatitis: A retrospective analysis of 77 cases
}

\author{
Attila Toth, Frederico Maria Guercini, Dawn Marta Feldthouse, Jun Chao Zhang \\ The MacLeod Laboratory, Weill-Cornell Medical College, New York Presbyterian Hospital, New York, US.
}

\begin{abstract}
Summary
Objective: To compare pre- and post-therapy symptom scores reported on the National

Institute of Health Chronic Prostatitis Symptom Index (NIHCPSI) after trans-rectal antibiotic injections therapy for men suffering from chronic prostatitis.

Materials and methods: Retrospective analysis of NIHCPSI symptom scores obtained from chart reviews of 77 treated males suffering from chronic prostatitis before and after trans rectal injections for the treatment of chronic prostatitis. Results: Most patients reported a $40 \%$ to $60 \%$ improvement in symptom scores. In subgroups comparing scores in patients with less than 5 injections, the improvement was less than in patients who received 10 or more injections. Patients' responses after a shorter (3 months) follow up showed better pain scores than patient's scores after longer, over one-year or more, follow-up periods.

Conclusion: Our findings show that direct antibiotic injection for chronic prostatitis is a viable addition to standard therapies. Improvements in symptom scores are long lasting. Discomfort is minimal and side effects are rare and avoidable.
\end{abstract}

KEY WORDS: Chronic prostatitis; Bacterial prostatitis; Injection therapy of prostatitis.

Submitted 14 October 2017; 17 December 2017

\section{INTRODUCTION}

Prostatitis accounts for approximately 2 million outpatient visits per year in the United States, including $8 \%$ of all visits to urologists and 1\% of those to primary care physicians. The direct costs of care approach $\$ 4,000$ per patient per year (1).

The clinical syndrome encompasses a wide range of male pelvic conditions from the well-defined bacterial prostatitis to the ill-defined chronic pelvic pain syndrome (CPPS). Only in about $5 \%$ of all cases is there a documented bacterial origin. Still, a very high percentage of patients receive antibiotic therapy at the first visit (2). About half of all patients presenting with prostatitis are in the reproductive age (3) and they have worse scores on questions related to mental and physical health-related quality of life issues than patients with congestive heart failure, diabetes mellitus or Crohn's disease $(4,5)$. It has been known for a long time that chronic prostatitis affects sperm quality, fertility and pregnancy rates (6). Symptomatic prostatitis and asymptomatic leucospermia make the treatment of infertility more challenging (7).
In response to failure of oral antibiotics to eradicate pathogens from the genital secretions of male patients being treated for infertility, we adopted direct antibiotic injections to the prostate and offered this treatment to patients for the last 17 years. Histology showing scarring, calcification, and sealed off bacteria in the prostate supported this approach. For the last 10 years, injection therapy has been also offered to patients suffering from symptoms of chronic prostatitis without infertility issues. This work aims to compare National Institute of Health Chronic Prostatitis Symptom Index (NIHCPSI) scores of patients suffering from prostatitis before and after injection therapy.

\section{Materials and Methods}

Eligible candidates for this retrospective study were 77 patients who presented to us for the treatment of prostatitis symptoms during a five-year period, between July 1 st, 2008 and July $1^{\text {st }}, 2013$. The mean duration of prostatitis in the study group was 83.91 and the range was 2-360 (in months). The mean age at first visit was 40.51 and ranged from 20-to 67 (in years).

The study was conducted with the approval of the Institutional Review Board at Weill Cornell Medical College - New York Presbyterian Hospital on 07/16/2014 (IRB Protocol Number: 130814262).

All patients had been given the diagnosis of chronic prostatitis by a urologist prior to our consultation. Except for a few patients presenting with previous cultures showing mostly enteric bacteria, all others' cultures were negative or not done, yet all had received courses of oral antibiotics prior. The patients signed a written informed consent for the injection therapy and were asked to fill out an NIHCPSI questionnaire and undergo physical and microbiological examinations prior to the start of the injection therapy. The patients were free to interrupt the injection therapy if they did not feel improvement of symptoms after the first two injections or anytime during the therapy course if there was no further improvement with subsequent injections.

Subgroups: Follow up NIHCPSI questionnaires were completed by 9 of the 77 patients within three months after the last injection, 29 subjects completed the questionnaire not more than 12 months and not less than three months following the completion of the therapy and for 38 patients the time for filling out the follow up questionnaire was longer than one year.

No conflict of interest declared. 
Office evaluation: The physical examination included manual and ultrasound evaluations of the prostate and seminal vesicles and taking of a urethral swab specimen for Chlamydia Direct Fluorescent Antibody (DFA) testing. Expressed Prostatic Secretion (EPS) or seminal fluid samples were used for microscopic examination and for bacteria cultures.

Microbiological examination of genital secretions: Chlamydia trachomatis was tested using the Pathfinder Direct Antigen Detection System from Bio Rad Laboratories. A7 differential agar was used to identify Mycoplasma, and Application Program Interface (API) systems were used for aerobic bacteria identification. Remel Rapid Ana (Anaerobic) II system was used to identify anaerobic bacteria. The API 20c AUX system (brand name from Biomerieux for yeast identification system) was used to identify yeast.

The Rapid NH (Neisseria Hemophilus) System identified Neisseria and Hemophilus.

Trichomonas vaginalis was identified by directly observing the fresh secretion for moving parasites with flagella. The result of the culture studies did not influence the recommendation for injection therapy.

Antibiotics selected for the injected cocktail: Based on a literature search we compiled a list of bacteria implicated in prostatitis and selected antibiotics with ample coverage for all that were listed. Six compatible antibiotics were mixed in a cocktail to be delivered in one injection. Each 10-ml volume of the cocktail contained the following antibiotics: gentamicin, $80 \mathrm{mg}$, clindamycin, 150 $\mathrm{mg}$, metronidazole, $10 \mathrm{mg}$, moxifloxacin, $3.2 \mathrm{mg}$, fluconazole, $2 \mathrm{mg}$ and azithromycin, $50 \mathrm{mg}$.

Methylprednisolone, $50 \mathrm{mg}$ was added to this mixture to activate intracellular dormant reticulate bodies of Chlamydia trachomatis. The azithromycin and methylprednisolone were reconstituted in $10 \mathrm{ml}$ of $1 \%$ lydocaine each. A standard endo-cavitary probe was equipped with a needle guide and a 22-gauge spinal needle was used to deliver $12 \mathrm{ml}$ of the cocktail per injections; $3 \mathrm{ml}$ each to the right and left seminal vesicles and to the right and left lobes of the prostate. One tablet of hydrocodone $5 \mathrm{mg} /$ acetaminophen $300 \mathrm{mg}$ with $800 \mathrm{mg}$ of ibuprofen were sufficient for both sedation and pain management.

\section{Statistical analysis}

Analysis compared pre-and post-therapy NIHCPSI scores and examined whether the magnitude of change in symptom scores after injection therapy related to any of the following: a) Duration of prostatitis in months, b) Age at first visit c) Chlamydia status at first visit d) Number of injections received; less than 5 injections, 5-10 injections, more than 10 injections, e) The difference in reported symptom changes in post-therapy intervals: Follow-up within 3 months, 3-12 months, 12 months or more.

SPSS v. 20 (8) was used for all descriptive and inferential analyses. A 95\% level of significance was set for all inferential tests. Inferential tests included paired t-tests, within groups analysis of covariance tests (ANCOVA) and mixed between-within groups analysis of variance tests (ANOVA).

Chance of benefiting from injection therapy were calculated comparing pre- and post-therapy dependent vari- ables (outcomes) for the inferential tests. The NIHCPSI scores were defined as the assessment measures for "benefiting from the injection therapy (or the extent of benefit)", and were classified into four outcomes of: Pain (scale of $0-22$ ), Urinary (scale of 0-10), Quality of Life (scale of 012), and Total Score (scale of 0-44). Higher numbers in the scores indicated more pain, more urinary problems and less quality of life, resulting in higher Total Scores. Questions to be answered were: Does the magnitude of change in symptom scores after injection therapy relate to any of the following categorical variables (Table 1): 1 . Duration of prostatitis (in months), 2. Age at first visit (in years), 3. Chlamydia status at first visit, 4. Number of injections received; less than 5 injections, 5-10 injections, more than 10 injections, 5. The difference in reported symptom changes in post-therapy intervals: Follow-up within 3 months, 3-12 months, 12 months or more.

\section{RESULTS}

Although, most study patients had been previously classified as suffering from "non-bacterial prostatitis", we recovered a variety of both aerobic and anaerobic bacteria from genital secretions of those who underwent testing. $80 \%$ of those tested were positive for Chlamydia trachomatis from the urethral swab specimens (Table 2).

Table 3 calculates the measures of central tendency for variables of the study and ranges of the categorical variables before and after intervention.

Preliminary tests performed a series of paired-sample ttests to compare the pre-and post-intervention NIHCPSI scores $(\mathrm{N}=77)$.

One ANCOVA was performed for each of the four NIHCPSI variables. For all four NIH symptom scores the t-test analysis showed significant pre-and post-therapy differences (Table 4).

The duration of prostatitis (in months) and influence of age at first visit were included as covariates for each of the four ANCOVA tests. There was no significant interaction effect found between the duration of prostatitis or the co-variates of age at first visit and any of the symptom scores or total NIHCPSI scores.

However, there was a significant main effect for time. The post-therapy NIHCPSI scores were significantly lower than the pre-treatment NIHCPSI scores for all variables $(\mathrm{p}<.05)$, and mirrored the findings of the paired t-test in Table 3. Although there were significant decreases in all four NIHCPSI variable outcomes over time there was no significant effect of age at first visit or the duration of prostatitis.

\section{Chlamydia status at first visit}

A series of four two-way ( 2 X 2) mixed between-within groups analysis of variance (ANOVA) tests were performed to answer question number three. Each of the four analyses included one repeated measures (withingroup) independent variable of time with 2 levels (preintervention and post-intervention), and one betweengroup independent variable of chlamydia status at first visit, with two categories (positive vs. negative). Again, time was statistically significant for all four NIH variable outcomes. All four NIHCPSI scores significantly 
Table 1.

Frequencies and percentages of the categorical variables of the study $(N=77)$.

\begin{tabular}{|lcc|}
\hline Categorical variables & Frequency & Percent \\
\hline Chlamydia status at first visit & 56 & 72.7 \\
Positive & 14 & 18.2 \\
Negative & 7 & 9.1 \\
Missing & 12 & \\
\hline Number of injections received & 15.6 \\
Less than 5 & 33 & 42.9 \\
5-10 & 32 & 41.6 \\
More than 10 & 9 & \\
\hline Post therapy follow-up intervals & 11.7 \\
Within 3 months & 29 & 37.7 \\
3-12 months & 38 & 49.4 \\
12 months or more & 1 & 1.3 \\
Missing & & \\
\hline Age at enrolment in the study (in years) Mean = 40.51, Range 20-67. & \\
Duration of prostatitis (in months) Mean = 83.91, Range 2-360. & \\
\hline
\end{tabular}

Table 2.

Shows the microbiological findings prior to initiating the injection therapy. A variety of aerobic and anaerobic bacteria were isolated and $80 \%$ of those tested showed Chlamydia trachomatis elementary bodies in the urethral swab specimen $(N=77)$.

\begin{tabular}{|lcccccccc|}
\hline Variable & $\begin{array}{c}\text { Number } \\
\text { tested }(\mathbf{n})\end{array}$ & \% of N & $\begin{array}{c}\text { Number } \\
\text { positive }\end{array}$ & $\begin{array}{c}\% \text { of } \\
\text { tested }\end{array}$ & $\begin{array}{c}\% \text { of } \\
\mathbf{N}\end{array}$ & $\begin{array}{c}\text { Number } \\
\text { negative }\end{array}$ & $\begin{array}{c}\% \text { of } \\
\text { tested }\end{array}$ & $\begin{array}{c}\% \text { of } \\
\mathbf{N}\end{array}$ \\
\hline Chlamydia & 70 & $91 \%$ & 56 & $80 \%$ & $73 \%$ & 14 & $20 \%$ & $18 \%$ \\
\hline Mycoplasma & 60 & $78 \%$ & 4 & $7 \%$ & $5 \%$ & 56 & $93 \%$ & $73 \%$ \\
\hline Aerobes & 61 & $79 \%$ & 41 & $67 \%$ & $53 \%$ & 20 & $33 \%$ & $26 \%$ \\
\hline Anaerobes & 61 & $79 \%$ & 52 & $85 \%$ & $68 \%$ & 9 & $15 \%$ & $12 \%$ \\
\hline
\end{tabular}

decreased $(p<.05)$ from pre-to post-intervention. However, there was no significant interaction or main effect of chlamydia status at first visit.

\section{Number of injections received}

A series of four two-way ( 3 X 2) mixed between-within groups analysis of variance (ANOVA) tests were performed to address this question. Each of the four analyses included one repeated measures (within-group) independent variable of time with 2 levels (pre-intervention and post-intervention), and one between-group independent variable of number of injections received, with three categories of: (a) less than $5(\mathrm{~N}=12)$, (b) 5$10(\mathrm{~N}=33)$, and (c) more than $10(\mathrm{~N}=32)$.

The dependent variables (outcomes) of interest were (a) pain, (b) urinary, (c) Quality of Life, and (d) total score. NIHCPSI Pain scores: In group 1 (less than 5 injections) there was no significant interaction effect found between Pain and the number of injections received, However, in patients who received between 5 and 10 injections, there was a significant main effect of number of injections received $[F(2.74)=4.351, p=0.016$, partial eta squared $=$.105]. A post hoc analysis via Tukey's Honestly Significant Difference test (HSD) indicated Group 2 (5-10 injections) showed a significantly lower Pain score $(\mathrm{M}=$ 2.667, SE $=1.076$ ) compared to Group 1 (less than 5 injections; $\mathrm{M}=8.99, \mathrm{SE}=.649 ; \mathrm{p}=.012$ ). Group 3 (more than 10 injections) did not differ significantly from group 2. There was also a significant main effect for the within-groups variable of time. This finding mirrors the findings of the paired t-test for Pain from Table 4.
NIHCPSI urinary scores: There was no significant interaction effects or main effects found for the number of injections received on the urinary scores. There was a significant effect for the within-groups variable of time. This finding mirrors the findings of the paired t-test for NIH Urinary from Table 4.

NIHCPSI Quality of Life scores: There was a significant interaction of the between-groups variable categories of the number of injections variable and the pre-and postintervention Quality of Life score. The Quality of Life score decreased more from pre-to post-intervention for those who received 5-10 injections or more than 10 injections, than for those who received less than 5 injections $(\mathrm{F}(2.74)=4.19, \mathrm{p}=.019)$.

Figure 1 is a graphical representation of the interaction effect. Tests of simple effects were performed to investigate the significant interaction effect. A series of three paired-samples t-tests were conducted to look at the difference between Quality of Life score pre-and post-therapy. One t-test was performed for each of the individual between group levels of (a) less than 5 injections (Group 1), (b) 5-10 injections (Group 2). and (c) more than 10 injections (Group 3). In Group 1 (less than 5 injections), there was no statistically significant difference in the Quality of Life score from preto post-therapy $[\mathrm{t}(11)=1.915$, $\mathrm{p}=.082]$. For Group 2 (5-10 injections) the pre-therapy Quality of Life Score ( $M=9.79$, SD = 2.233) was significantly higher compared to the post-therapy score $[\mathrm{M}=$ $5.18, \mathrm{SD}=3.21 ; \mathrm{t} \mathrm{32})=8.22, \mathrm{p}<0.005]$

For Group 3 (more than 10 injections) the pre-therapy Quality of Life score was significantly higher $(\mathrm{M}=9.94$, $\mathrm{SD}=1.90$ ) than the post-therapy Quality of Life score [M $=5.47, \mathrm{SD}=2.95 ; \mathrm{t}(31)=6.901, \mathrm{p}<0.005]$.

Figure 1.

Graph shows significant difference between the number of injections and the pre- and post-therapy NIHCPSI Quality of Life scores. In Groups 2 and 3 the NIHCPSI Quality of Life scores significantly decreased from pre- to posttherapy. Though Group 1 also decreased in scores, the decrease however was not significant.

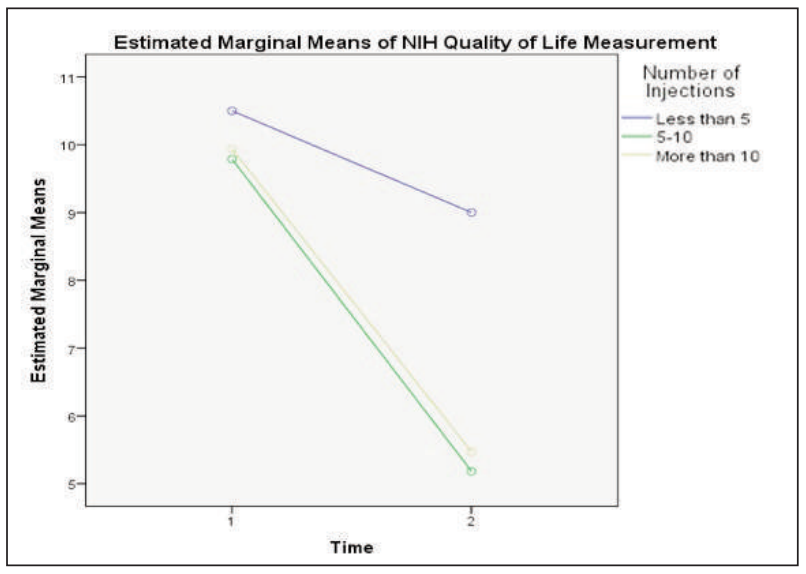


Figure 2.

Graph shows significant between-groups differences as to the length of time to follow up. NIHCPSI improvement in Pain Score diminished after longer follow up.

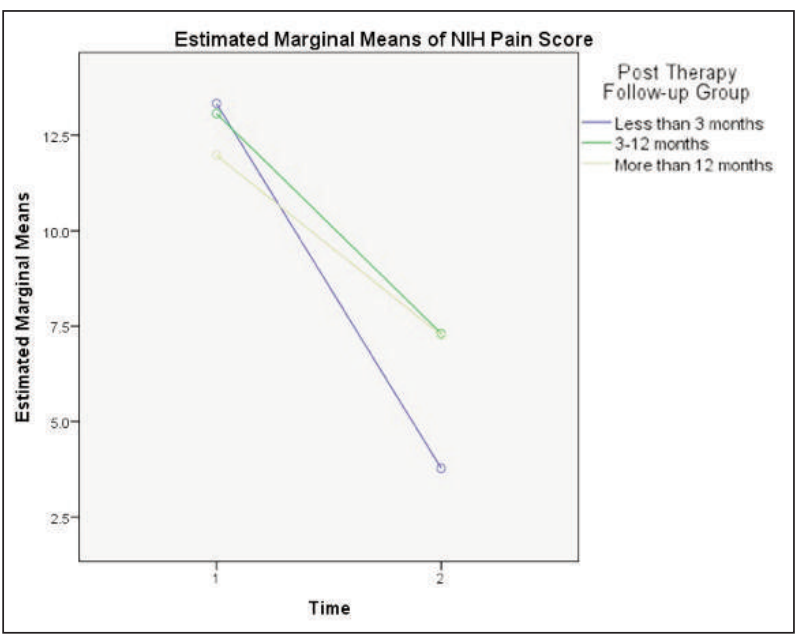

Figure 3.

Histogram showing the distribution of the percent improvemnet in NIHCPSI Total Scores. $60 \%$ of the treated patients reported at least $50 \%$ improvement.

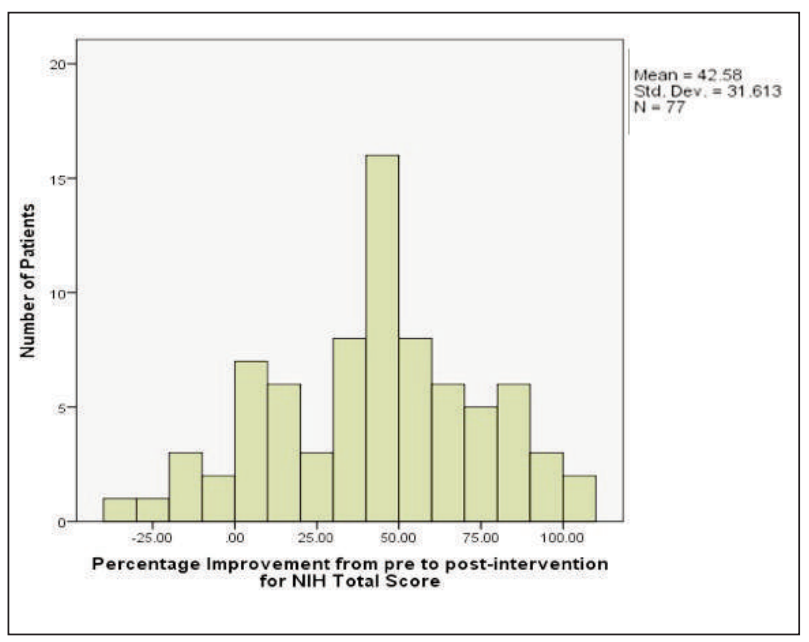

number of injections received $[F(2,74)=6.902, \mathrm{p}=$ 0.002 , partial eta squared $=.157]$. A post hoc analysis via Tukey's HSD indicated that the NIHCPSI Total scores for Group 1 (less than 5 injections; $M=27.708, S E=1.711$ ) were significantly greater than Group 2 (5-10 injections; $\mathrm{M}=20.424, \mathrm{SE}=1.032 ; \mathrm{p}=.001$ ) and Group 3 (more than 10 injections; $\mathrm{M}=21.391, \mathrm{SE}=1.048 ; \mathrm{p}=.007$ ). There was also a significant main effect for the withingroups variable of time. The NIHCPSI Total Scores at post-intervention $(\mathrm{M}=15.83, \mathrm{SD}=8.979)$ were significantly lower than the total pre-intervention NIHCPSI Total Scores $[\mathrm{M}=28.09, \mathrm{SD}=6.775 ; \mathrm{F}(1.74)=87.544$, $\mathrm{p}<0.0005$, partial eta squared $=0.542]$. This finding mirrors the findings of the paired t-test for NIHCPSI Total Score from Table 4.

In conclusion, there were significant decreases in all four NIHCPSI variable outcomes from pre- to post-intervention. Additionally, the mean value of Total Score was significantly higher for Group 1 than for Groups 2 or 3.

\section{Time to follow up}

A series of four two-way ( 3 X 2) mixed between-within groups analysis of variance (ANOVA) tests were performed to the lapse of time until follow up. Each of the four analyses included one repeated measures (withingroup) independent variable of time with 2 levels (pre-

Table 3.

Measures of central tendency for the four NIHCPSI variables of the study $(N=77)$.

\begin{tabular}{|lcccc|}
\hline Variable & $\boldsymbol{M}$ & SD & Mdn & Range \\
\hline NIH pain & & & & \\
Pre-test & 12.66 & 4.35 & 13 & $0-21$ \\
Post-test & 7.03 & 4.944 & 7 & $0-19$ \\
\hline NIH urinary & & & & \\
$\quad$ Pre-test & 5.47 & 2.950 & 6 & $1-10$ \\
$\quad$ Post-test & 2.91 & 2.586 & 2 & $1-10$ \\
\hline NIH Quality of Life & & & & \\
$\quad$ Pre-test & 9.96 & 2.029 & 10 & $2-12$ \\
$\quad$ Post-test & 5.90 & 3.267 & 6 & $0-12$ \\
\hline NIH total & & & & \\
$\quad$ Pre-test & 28.09 & 6.775 & 28 & $14-43$ \\
$\quad$ Post-test & 15.83 & 8.979 & 15 & $0-38$ \\
\hline M = Mean; SD = Standard Deviation; Mdn = Median. & \\
\hline
\end{tabular}

In summary, Group 2 (5-10 injections) and Group 3 (more than 10 injections) showed a significant improvement in the NIHCPSI Quality of Life scores from pre-to post-intervention. However, Group 1 (less than 5 injections) did not.

NIHCPSI Total score: A mixedmodel ANOVA was conducted to test the pre-and post-intervention scores for Total score, with the between-subjects variable of number of injections received and the within-subjects variable of time. There was a significant main effect for the between-subjects variable,
Table 4.

Descriptive Statistics and Results of Paired Samples T-Tests for Study

Outcomes (performed for the four NIHCPSI scores with highly significant $p$ values for the difference between pre-intervention to post-intervention scores $(N=77)$.

\begin{tabular}{|c|c|c|c|c|c|c|c|c|c|}
\hline Variable/time & M & $S D$ & Mdn & Range & $\begin{array}{l}\text { Mean } \\
\text { Diff. }\end{array}$ & $\begin{array}{c}\text { SE Mean } \\
\text { Diff. }\end{array}$ & $t$ & p & $\eta^{2}$ \\
\hline NIH Pain & & & & & 5.63 & 0.59 & 9.63 & $<.0005$ & 0.38 \\
\hline Pre-test & 12.66 & 4.35 & 13 & $0-21$ & & & & & \\
\hline Post-test & 7.03 & 4.94 & 7 & $0-19$ & & & & & \\
\hline NIH Urinary & & & & & 2.56 & 0.31 & 8.19 & $<.0005$ & 0.31 \\
\hline Pre & 5.47 & 2.95 & 6 & $1-10$ & & & & & \\
\hline Post & 2.91 & 2.59 & 2 & $1-10$ & & & & & \\
\hline NIH Quality of life & & & & & 7.05 & 0.35 & 20.02 & $<.0005$ & 0.73 \\
\hline Pre & 9.96 & 2.03 & 10 & $2-12$ & & & & & \\
\hline Post & 5.90 & 3.26 & 6 & $0-12$ & & & & & \\
\hline NIH Total & & & & & 12.26 & 1.09 & 11.29 & $<.0005$ & 0.46 \\
\hline Pre & 28.09 & 6.78 & 28 & $14-43$ & & & & & \\
\hline Post & 15.83 & 8.98 & 15 & $0-38$ & & & & & \\
\hline
\end{tabular}


intervention and post-intervention), and one betweengroup independent variable of number of injections received, with three categories of: (a) less than 3 months $(\mathrm{N}=9)$, (b) 3-11 months $(\mathrm{N}=29)$, and (c) 12 or more months $(\mathrm{N}=38)$. The dependent variables (outcomes) of interest were (a) pain, (b) urinary, (c) Quality of Life, and (d) total.

NIHCPS Pain scores: There was a significant interaction of the between-groups variable categories of the time to follow up variable and the pre- and post-intervention Pain score. Pain score decreased more for those whose follow up was at the less than 3 months level, than for the other two follow up groups $(\mathrm{F}(2.73)=3.43, \mathrm{p}=.038)$ (Figure 2). Tests of simple effects were performed to investigate the significant interaction effect. A series of three paired-samples t-tests were conducted to look at the difference between Pain score pre-and post- therapy intervention. One t-test was performed for each of the individual between group levels of (a) less than 3 months (Group 1), (b) 3-11 months (Group 2), and (c) 12 or more months (Group 3). Group 1 (less than 3 months): There was a statistically significant mean difference from the pre-intervention score $(\mathrm{M}=13.33, \mathrm{SD}=6.403)$ to the post-intervention score $[\mathrm{M}=3.78, \mathrm{SD}=4.236$; $\mathrm{t}$ (28) $=7.373, \mathrm{p}<0.0005]$. Group 2 (3-11 months): There was a statistically significant mean difference from the preintervention score ( $\mathrm{M}=13.07, \mathrm{SD}=2.963)$ to the postintervention score $[\mathrm{M}=7.31, \mathrm{SD}=4.401, \mathrm{t}(37)=5.415$, $\mathrm{p}<0.0005]$. Group 3 (12 or more months): There was a statistically significant mean difference from the preintervention score $(\mathrm{M}=11.97, \mathrm{SD}=4.571)$ to the postintervention scores $[\mathrm{M}=4.26, \mathrm{SD}=5.012, \mathrm{t}(37)=$ 5.415, $\mathrm{p}<0.005]$. All three times to follow-up groups had significant decreases in the mean Pain scores, but Group 1 indicated a larger decrease from pre-to postintervention. NIHCP.

SI urinary scores: There was no significant interaction effect found between NIH Urinary from pre- to postintervention and time to follow up or for the main effect of time to follow-up $(p>.05)$. There was a significant effect for the within-groups variable of time. The total post-test Urinary scores were significantly lower than the pre-test NIH Urinary scores $(\mathrm{p}<.05)$. This finding mirrors the findings of the paired t-test from Table 4.

NIHCPSI Quality of Life: A mixed-model ANOVA was conducted to test the pre- and post-intervention scores for NIH Quality of Life with the between-subjects variable of time to follow up and the within-subjects variable of time. There was a significant effect for the withingroups variable of time. The total post-therapy NIH Quality of Life scores $(\mathrm{p}<.05)$. This finding mirrors the findings of the paired t-test for NIH Quality of Life scores from Table 4.

NIHCPSI Total scores; A mixed-model ANOVA was conducted to test the pre-and post-intervention scores for Total score with the between-subjects variable of time to follow up and the within-subjects variable of time. There was a significant effect for the within-groups variable of time. The mean post-therapy Total scores were significantly lower than the mean pre-therapy scores $(\mathrm{p}<.05)$. This finding mirrors the findings of the paired t-test in Table 4.

\section{Discussion}

Until very recently it was a commonly accepted paradigm that chronic bacterial prostatitis is a rare condition, representing 3\% to $10 \%$ of all cases of chronic prostatitis ( 9 , 10). Long term antibiotics therapies are still widely administered, and patients report moderate to marked improvement (11). The use of the polymerase chain reaction (PCR) to diagnose bacterial infections in men with prostatitis (49\% for Chlamydia) moved a significant \% of patient from non- bacterial to bacterial prostatitis (12). In our series of 77 patients, among those tested, finding of Chlamydia trachomatis elementary bodies was $80 \%$. This higher isolation rate suggests that in chronic infections the yield for extracellular Chlamydia forms diminishes in commonly tested genital fluid samples (urine, semen or EPS) and epithelial tissue samples (urethral swab, prostate biopsy) will help identify slow growing intracellular forms more readily (13). In addition to Chlamydia we found a variety of aerobic and anaerobic bacteria in semen or EPS samples suspected of contributing to both local immune responses and to tissue damage associated with Chlamydia infection (14). Though a subgroup analysis did not show influence of the Chlamydia isolation rate and the chance of benefiting from the injection therapy, it is tempting to speculate that the frequently noted exacerbation of symptoms during prostatitis is best understood by following the intra and extra-cellular phases of the Chlamydia life cycle. The frustration with repeatedly failed single antibiotic courses could best be explained by the development of resistant Chlamydia strains to the single antibiotic. We propose that the frequently seen hyaline containing scarred, and later calcified nodules, often seen in chronic prostatitis, represent local tissue reaction to Chlamydia antigens with simultaneous entrapment of Chlamydia and other bacteria.

A reduced blood supply to these areas makes it difficult to achieve therapeutic concentration of antibiotics in such areas (15). Even if some antibiotics penetrate the cell wall they will have little effect on the viral, reticulate form of Chlamydia. The rationale behind adding steroids to the antibiotic cocktail is the ability of steroids to enter the cell and activate dormant, altered forms (spore forms) of Chlamydia. By keeping antibiotics in the extracellular space long enough, a gradual depletion of the Chlamydia bacterium can be accomplished. This could explain, at least in part, the inferior response in Quality of Life scores of patients with five or less injections compared to the other two groups, between 5 and 10, or more than 10 injections groups.

Histology of the chronically infected prostate showing scarring, calcification, and sealed off bacteria supports injection therapy (16). In some cases, as late as six months to a year following injection therapy we have seen a recurrence of symptoms and offered booster injections that were successful in such cases. Residual, recurring, or re-infection could be the explanation for reduced symptom improvement that was reported after the longer follow ups.

Orally given multi-drug regimens are poorly tolerated. By mixing six compatible antibiotics in one cocktail and injecting them trans rectally, one can safely reach thousand-fold the tissue concentration than the one reached 
by a single orally administered antibiotic. In our experience, injection therapy is safe, well tolerated and all complications are minor including the physical discomfort, pain on injection, minimal bleeding in the semen, urine or stool. No local infection, abscess formation or trauma complicated any of several hundreds of injections administered by our clinic.

This study is retrospective, and we recognize the limitations. We believe however that the information contained in this study is significant enough to encourage the urology community to adopt injection therapy as a viable therapy for chronic prostatitis.

\section{ACKNOWLEDGEMENTS}

The Authors wish to thank Ms. Elaine Eisenbeisz (Statistician, Omega Statistics, 40960 California Oaks Road, Suite 215, Murrieta, CA 92562 http://www.omegastatistics.com) for review and analysis of data and Dr. Yu-Xin Liu (Microbiologist, for the microbiological testing of genital secretions. 40-35 Ithaca Street Apt 5E, Elmhurst, NY 11373, yuxinliu89@hotmail.com).

\section{References}

1. Schaeffer AJ. Chronic Prostatitis and the Chronic Pelvic Pain Syndrome. N Engl J Med. 2006; 355:1690.

2. Collins M, Fowler F, Elliott D, et al. Diagnosis and treating chronic prostatitis: Do urologist use the four-glass test? Urology. 2000; 55:403.

3. Collins M, Stafford R, O'Leary M, Barry M. How common is prostatitis? A national survey of physician visits. J Urol. 1998; 159:1224.

4. Wenninger K, Heiman J, Rothman I, et al. Sickness, impact of chronic nonbacterial prostatitis and its correlates. J Urol. 1996; 155:965.
5. McNaughton Collins M, Pontari MA, O'Leary MP, et al. Quality of life is impaired in men with chronic prostatitis: Chronic Prostatitis Collaborative Research Network. J Gen Inter Med. 2001; 16:656.

6. Weidner W, Krause W, Ludwig M. Relevance of male accessory gland infection for subsequent fertility with special focus on prostatitis. Human Reprod Update. 1999; 5:421.

7. Giamarellou H, Tympanidis K, Bitos NA, et al. Infertility and chronic prostatitis. Andrologia 1984; 16:417.

8. Cohen J. Statistical Power Analysis in Directions of Psychological Science, Sage Publications, Inc. 1992; Vol. 1, No. 3, p.98-101.

9. Lipsky, Benjamin A, Byren I, Byren H, Christopher T. Treatment of bacterial prostatitis. Reviews of Anti-Infective Agents. 2010; 50:1641.

10. Orland SM, Hanno PM, Wein AJ. Prostatitis, prostatosis and prostatodynia. Urology. 1985; 25:439.

11. Nickel JC, Downey J, Johnston B, Clark J. Predictors of patient response to antibiotic therapy for the chronic prostatitis/chronic pelvic pain syndrome: a prospective multicenter clinical trial. J Urol. 2001; 165:1544.

12. Choi YS, Kim KS, Choi SW, et al. Microbiological etiology of bacterial prostatitis in general hospital and primary care clinic in Korea. Prostate Int. 2013; 1:133.

13. Stephens RS. The cellular paradigm of chlamydial pathogenesis. Trends Microbiol. 2003; 11:44.

14. Hu VH, Weiss HA, Ramadhani AM, et al. Innate immune responses and modified extracellular matrix regulation characterize bacterial infection and cellular/connective tissue changes in scarring trachoma. Infect Immune. 2012; 80:121.

15. Darville T, Hiltke TJ. Pathogenesis of genital tract disease due to Chlamydia trachomatis. J Infect Dis. 2010; 201 (Suppl. 2): S114.

16. Madsen PO, Baumueller A, Hoyme U. Experimental models for determination of antimicrobials in prostatic tissue, interstitial fluid and secretion. Scand J Infect Dis Supl. 1978; 14:145.

\section{Correspondence}

Attila Toth, MD (Corresponding Author)

TothMD@gmail.com

Director, MacLeod Laboratory, Associate Clinical Professor,

New York Presbyterian Medical Center

New York Office: 65 East $79^{\text {th }}$ Street, New York, NY. 10075

Florida Office: 3893 Military Trail, Suite 1. Jupiter, FL 33458 (surface correspondence)

http://www.fertilitysolution.com

Frederico Maria Guercini, MD

Professor of Urology

Via della Camilluccia 600, 00135 Rome, Italy

guercini@mclink.il

Dawn Marta Feldthouse

dawn.feldthouse@gmail.com

Staff Nurse, The MacLeod Laboratory

Jun Chao Zhang

juneczhang17@gmail.com

Pre-Med Student, Syracuse University, US 